\title{
In Memory of Professor Shoshichi Kobayashi
}

\section{by Shing-Tung Yau}

I am grateful to Professor Ochiai for inviting me to attend the conference in memory of Professor Kobayashi.

I was shocked last year to learn that Professor Kobayashi had passed away. I thought he was still young and full of energy. Professor $\mathrm{H}$. Wu told me that he passed away peacefully when he was traveling on the airplane back from Japan to Berkeley. Instantly, I recalled his smile when I met him first time as a student in Berkeley. I learnt a great deal from his seminar in differential geometry which he and Prof. Ochiai organized.

A very touching piece of information that I learnt in this trip to Japan is what Professor Ochiai told me: Professor Kobayashi was the chairman of the admission committee for the mathematics department in Berkeley in the year I applied to be a graduate student in Berkeley. For many years, I knew the roles played by Professor Donald Sarason and Professor S. S. Chern in my admission to Berkeley, but I did not realize that Professor Kobayashi had also played a critical role. And he kept this information secret from me in his whole life. Professor Ochiai told me that he pushed strongly to give me the best that Berkeley could offer. Indeed, this was the case as I was offered the prestigious IBM fellowship that year. I was offered an annual fellowship of $\$ 3000$, which was above the $\$ 2400$ that an American student would get in those days. Professor Ochiai said that Professor Kobayashi had told him that he considered admitting me to Berkeley to be one of his major achievements. I am truly grateful for his kindness.

At the time I was applying to Berkeley, I was a third year undergraduate student in Hong Kong and did not have a Bachelor degree from the Chinese University of Hong Kong. On the advice of Professor Salaff in Hong Kong, I only applied to one graduate school. My admission to Berkeley was decisive for my life and my mathematical career. The fellowship meant a great deal to me and my family. My father passed away very early and my family was poor. After I received the IBM fellowship, I sent half of the income back home and the financial problem in my family was greatly relieved. More importantly, I learnt the development of modern mathematics in Berkeley, which was the foundation of my later development in mathematics.

I was interested in functional analysis in Hong Kong. After taking and auditing several courses in Berkeley, I started to realize the beauty of geometry. The seminars ran by Professors Kobayashi and Ochiai have been particularly helpful. In order to follow their seminars, I spent a great deal of time to study the book by Hirzebruch: Topological Methods in Algebraic Geometry. That was an important turning point for me. Although I had asked Professor Chern to be my advisor, he was on sabbatical leave in my first year of graduate school. What I ended up learning about the foundation of geometry and topology

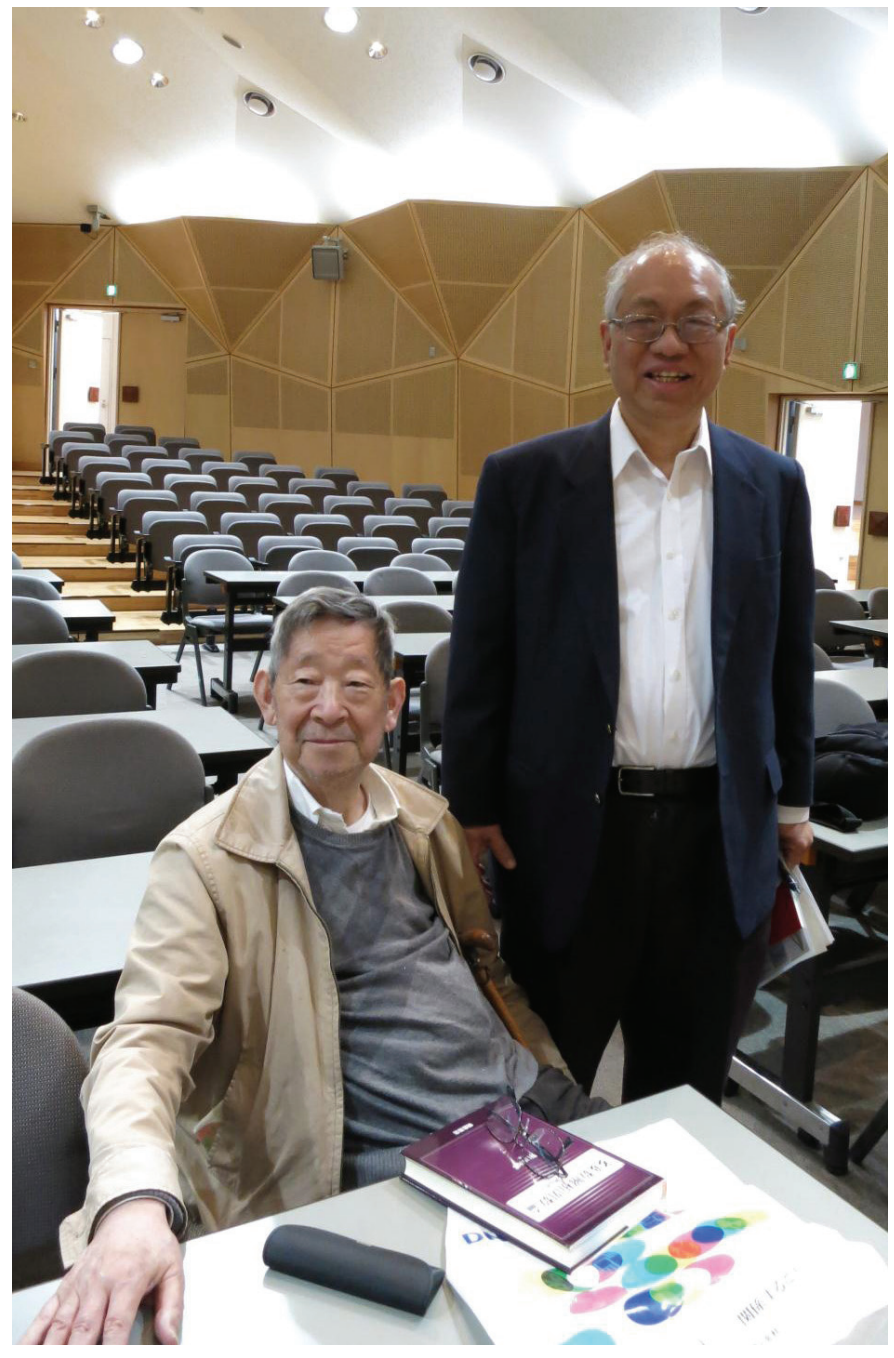

Masatake Kuranishi (Prof. Emeritus, Columbia University) and Shing-Tung Yau at the Memorial Symposium for Prof. Shoshichi Kobayashi, 25 May, 2013.

was from the lectures of Professor Kobayashi, along with those of Blaine Lawson and Ed Spanier.

In the spring of 1970, Professor Kobayashi had just finished the writing of his famous book on hyperbolic manifolds. I was very touched that he gave me the whole manuscript before it appeared in print. It is a very elegantly written book, I learnt the important Schwarz-Pick lemma from Chern's paper and from this book. I thought 
about this lemma a lot. I found its real analogue in the form of a gradient estimate, and this technique influenced most of my works in partial differential equations: the Li-Yau inequality came from understanding the parabolic version of this estimate. My second order estimate in the Calabi conjecture was also inspired by this lemma. I also made an effort to generalize the intrinsic measure introduced by Kobayashi and his student Eisenmann, and eventually I was able to turn it into a birational invariant measure.

In the year 1970 to 1971 , Kobayashi and Ochiai made an attempt to solve the Frankel conjecture which says that a compact Kähler manifold with positive bisectional curvature is biholomorphic to the complex projective space. Two important techniques were used. One is a vanishing theorem due to Bishop and Goldberg on proving that the second Betti number is equal to one. The other one is the existence of a rational curve with minimal degree, which goes back to the works of Hirzebruch and Kodaira. Their application of the Riemann-Roch formula and vanishing theorem greatly impressed me. In 1978, I told Siu about the Frankel conjecture and these ideas of Kobayashi-Ochiai. I suggested that an effective method to produce rational curves would be to prove area minimizing minimal spheres are holomorphic or antiholomorphic. Then we applied the newly developed theory of minimal sphere of Sacks-Uhlenbeck to demonstrate the existence of rational curves with minimal degree. We succeeded in proving the Frankel conjecture while at the same time, Mori proved the Hartshorne conjecture which is stronger. The idea of using minimal surface to produce rational curves of minimal degree was taken up by Gromov to develop pseudo holomorphic curves in symplectic geometry.

After I got the Fields Medal in 1982, I was told that Professor Kobayashi kindly took on the task of writing an article to describe my works in Japanese. We shared many ideas in geometry, especially those on the stability of bundles, after noting the work of Bogomolov on the Chern number inequality.

In addition to mathematics, Professor Kobayashi treated me very nicely as a student and as a friend. I stayed in his home briefly when I visited Bonn in 1978, before the International Congress in Helsinki. In those days, Professor Kobayashi was serving as chairman of the Berkeley department. His leadership is now described by his colleagues as "stellar" and "heroic." His smile and his diplomatic approach to the dean enabled him to get the dean to grant the department many of its wishes. His warm hospitality for the department's visitors from all over the world, especially those at the beginning of their careers, was well-known to all. We will certainly always remember his contributions to mathematics and to the mathematics community

\section{Appendix}

At the Memorial Reception for Professor Shoshichi Kobayashi on 25 May 2013, I read a Chinese poem that I wrote in dedication to Professor Kobayashi:

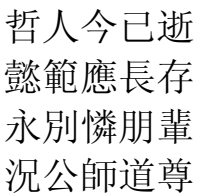

Dr. Donald T. Tang, a friend of Prof. Hisashi Kobayashi (Shoshichi's younger brother) has translated this poem into English:

The wise scholar has now passed away, Yet his excellent style remains forever, At this farewell I miss him as a close friend, And furthermore as an honorable and respected teacher. 